\begin{tabular}{cc} 
Sharif University of Technology \\
Scientia Iranica \\
SCIENTIA & Transactions F: Nanotechnology \\
I RAN I CA & \\
\hline
\end{tabular}

\title{
A biosensor based on plasmonic wave excitation with diffractive grating structure
}

\author{
V. Faramarzi, V. Ahmadi*, F. Ghane Golmohamadi and B. Fotouhi \\ Department of Electrical \& Computer Engineering, Tarbiat Modares University, Tehran, Iran.
}

Received 10 July 2016; received in revised form 6 February 2017; accepted 13 June 2017

\author{
KEYWORDS \\ Plasmonic device; \\ Refractive index; \\ Diffractive grating; \\ Surface plasmon \\ resonant; \\ Graphene; \\ Biosensing \\ application.
}

\begin{abstract}
We propose an active plasmonic device based on graphene. By using a diffractive grating on silicon, highly confined plasmonic waves in monolayer graphene are efficiently excited. The high electric field of the surface plasmons near the graphene layer makes them ideal for use in biosensors, where a very small change in refractive index should be detected. A small change in the refractive index of surroundings medium induces a large change in resonant wavelength. The spectral shift per refractive index unit $\left(\Delta \lambda_{0} / \mathrm{RIU}\right)$ of the sensor is considerable and exceeds $2000 \mathrm{~nm} / \mathrm{RIU}$ with a narrow spectral line width of the plasmon resonances. The figure of merit for this sensor is approximately equal to 14.2 and electrical tuning can be achieved by adjusting the Fermi level in graphene. Therefore, this grating plasmonic structure is highly sensitive and can be used in high-resolution biosensing applications.
\end{abstract}

(C) 2017 Sharif University of Technology. All rights reserved.

\section{Introduction}

Good electrical conductivity and mechanical strength, ultra-high mobility of massless fermions, relatively good transparency, high thermal conductivity, and high flexibility make graphene the best option for replacement or simultaneous use in the silicon world. From the viewpoint of optics, graphene has linear dispersion relationship, strong interaction with electromagnetic field, and high-speed response [1]. The distinctive characteristics of graphene make it suitable to propose integrated active plasmonic devices with a wide range of wavelengths from near-infrared to $\mathrm{THz}[2,3]$. By using the active plasmons, confined light can be manipulated and externally controlled in a structure with subwavelength dimensions [4]. Plasmonic waves

\footnotetext{
*. Corresponding author. Tel/Fax: +982182883368
} E-mail address: v_ahmadi@modares.ac.ir (V. Ahmadi)

doi: $10.24200 /$ sci. 2017.4253 can be strongly controlled with a broad adjustability range because plasmonic properties change dramatically when the free carrier density in a semiconductor is adjusted by several orders of magnitude. The carrier mobilities in conventional semiconductors are too low at room temperature, and cannot support plasmonic waves for acceptable propagation distances. However, Carrier mobility in graphene is high even at room temperature. Thus, plasmonic wave in the graphene film can be highly confined and propagate up to several micrometers [3]. In addition to broad adjustability, the electric field of a plasmonic wave is highly confined and localized in the thin layer of graphene. The highly localized optical field makes strong light-matter interactions and can be used to build Surface Plasmon Resonance (SPR) biosensors with high sensitivity [5].

Surface Plasmon Resonance (SPR) biosensors are optical sensors, which use surface plasmon waves to probe the interactions between biomolecules and the sensor surface. The technique of Surface Plasmon Resonant (SPR) is very sensitive to refractive index 
variations in sensing medium. When the liquid sample makes contact with the sensor surface, due to binding of analyte with affinity antibodies, which are immobilized on the sensor surface, the change in the plasmon resonant modes occurs. Therefore, material, which is in contact with the liquid sample (top layer of sensor), is influential and important in terms of refractive index and thickness of the adsorbed biolayer [6,7]. Recently, the graphene layer has been used for anchoring antibodies to the SPR sensor as new surface functionalization. The biomolecules are efficiently adsorbed on the graphene layer surface, due to the $\pi$-stacking interactions between graphene's hexagonal cells and the carbon-based ring structures in biomolecules [8,9]. Thus, the molecules get adsorbed on graphene surface, as the sample-containing analyte molecules come onto the graphene surface. This results in the formation of a layer of which the refractive index is higher than a buffer solution (water) and thus, it affects the resonant wavelength of plasmonic wave. As the performance of SPR sensor depends on the biomolecular adsorption, the material type of upper surface which immobilizes the biomolecules (antibodies) on the sensor surface plays an important role. In [10], we have proposed and analyzed new plasmonic-based structures for sequencing DNA molecules and detecting nanoscale objects by altering the external electromagnetic radiation to strong local field of the plasmon.

A key challenge in the plasmonic resonance sensors is coupling the surface plasmon with optical wave in free space, because plasmonic wave vector is much larger than free-space wave vector, which gives rise to the momentum mismatch between light and surface plasmon [11]. By using different coupler configurations such as prism couplers and grating couplers [12], the momentum of light and surface plasmon can be matched. In this work, we use rectangular diffractive grating to create plasmonic wave resonance in a monolayer graphene film. These resonant modes couple incident light to the Surface Plasmon Polariton (SPP) with maximum coupling efficiency and induce intense change in the optical transmission at the resonant frequency. The ability of resonant waveguide grating biosensors to monitor changes of cell adhesion of live cells in real time and label-free investigations of binding events makes it an attractive tool in medical application [13]. In [1416], plasmonic biosensors based on the graphene have been investigated. In these configurations, the prism coupler (Kretchmann configuration) has been used to excite the surface plasmon waves. However, diffraction grating structures are generally more compact, physically lighter, and less costly than the prism-coupled configurations. Therefore, for integrated biosensor design, grating-coupled SPR may be more beneficial as it allows the use of semiconductor materials as substrates. Another advantage of this coupling method over the previously mentioned methods is good reproducibility of coupling conditions since no further optical elements are directly involved.

We show that the diffractive grating device acts as a highly tunable biosensor that can be tuned with a small change in the Fermi level of graphene. By adjusting the Fermi level of graphene and grating periods, this structure can be used to build extremely sensitive biosensor with broad range of wavelengths from mid-infrared (mid-IR) to terahertz (THz).

\section{Materials and methods}

To excite SPP waves in the graphene, wave vector of incident light and SSP have to match. The use of optical gratings can compensate for the wave vector mismatching. Here, we use a silicon diffractive grating underneath the graphene, as shown in Figure 1, to excite the surface plasmons.

The effective refractive index of plasmonic mode is high and defined as $n_{\text {eff }}=\operatorname{Re}(\beta(\omega)) / k_{0}$ in the midIR wavelength range, where $\beta(\omega)$ is the in-plane wave vector in graphene. The grating period, $T$, which is related to frequency, $\omega_{0}$, is determined by the phase match equation $[4,17]$ :

$$
\operatorname{Re}\left(\beta\left(\omega_{0}\right)\right)-\frac{\omega_{0}}{c} \sin \theta=\frac{2 \pi}{T},
$$

where $c$ is the speed of light, $\theta$ is the incident angle, and $\omega_{0} / c$ is the vacuum wave vector, $k_{0}$. Under the normal-incidence wave condition $(\theta=0)$, a GuidedWave Resonant (GWR) is excited when the grating period, $T$, matches the period of the plasmonic wave $(2 \pi(\operatorname{Re}(\beta(\omega))))$. When the SPP waves are excited and propagated in graphene, optical energy is dissipated due to the ohmic loss in the graphene layer. Therefore, a notch can be seen in the transmission spectrum around the resonant frequency, $\omega_{0}$, as:

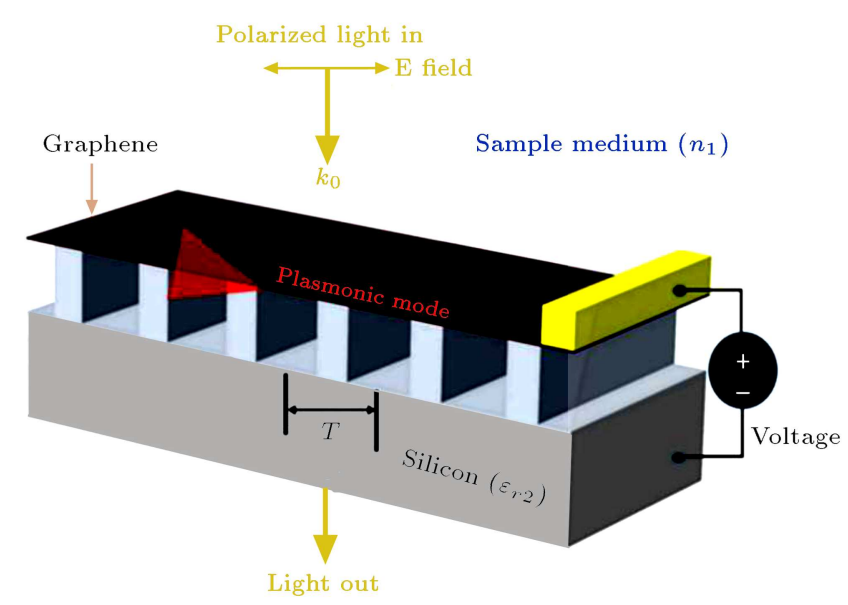

Figure 1. Schematics of a silicon diffractive grating based on plasmonic waves in the graphene layer. 


$$
\omega_{0}=\sqrt{\frac{2 e^{2} \times E_{f}}{\hbar^{2} \varepsilon_{0}\left(\varepsilon_{r 1}+\varepsilon_{r 2}\right) T}}
$$

where $\hbar$ is the reduced Planck constant, $\varepsilon_{0}$ is the vacuum permittivity, $\varepsilon_{r 1}$ and $\varepsilon_{r 2}$ are the dielectric constants of the materials above and below the graphene film, respectively, and $E_{f}=\hbar v_{f}(\pi n)^{1 / 2}$ is the Fermi energy level, where $n$ is the carrier density and $v_{f} \approx$ $10^{6} \mathrm{~m} / \mathrm{s}$ is the Fermi velocity in graphene [4,17]. To simulate the proposed structure, the finite element method is used. The graphene film is considered to be monolayer with the thickness of $t=0.5 \mathrm{~nm}$ and carrier mobility of $\mu \approx 10000 \mathrm{~cm}^{2} /$ (V.s). Moreover, the dielectric tensor components are $\varepsilon_{r 11}=\varepsilon_{r 22}=$ $2.5+i \sigma /\left(\varepsilon_{0} \omega t\right)$, where $\sigma$ is the conductivity of graphene, and the surface-normal component is set as $\varepsilon_{r 33}=2.5$ based on the dielectric constant of graphite.

The intraband conductivity of graphene at midIR frequencies is modelled with a semiclassical Drude model as:

$$
\sigma(\omega)=\frac{2 e^{2}}{\pi \hbar^{2}} k_{B} T_{k} \times \ln \left[2 \times \cosh \left(\frac{E_{f}}{2 k_{B} T_{k}}\right)\right] \frac{i}{\omega+i \tau^{-1}}
$$

where $T_{k}$ is temperature and $k_{B}$ is the Boltzmann constant. Here, the effect from the interband transition is neglected because the photon energy in the simulated spectral range is always less than $2 E_{f}$ [18].

\section{Results and discussion}

The evanescent field of the plasmonic wave, typically extending 30-100 $\mathrm{nm}$ into the antibody-analyte (biolayer) and cover solution layer, is sensitive to two types of ambient changes. The first one is altering the cover solution (a liquid sample that covers the sensing region) refractive index $\left(n_{1}\right)$. The second type is adsorption of molecules from a liquid sample on the surface of the sensing layer and formation of a thin adhesion layer with a specific thickness and refractive index.

The simulated normal-incidence transmission spectra with different ambient refractive indices are shown in Figure 2. As shown in the figure, there is a red shift at the resonant wavelength of spectra by changing the refractive index surrounding the graphene layer and diffractive grating $\left(n_{1}\right)$. This change may be caused by changes in biomolecules concentration, which in turn can produce a local change in the refractive index. According to the equation $\varepsilon_{r 1}=\left(n_{1}-i k_{1}\right)^{2}$, by increasing the refractive index of cover medium, the dielectric constant of the surrounding medium increases. The dielectric constant change in turn leads to a change in the propagation constant of plasmonic wave and will eventually lead to red shift in SPP resonant wavelength.

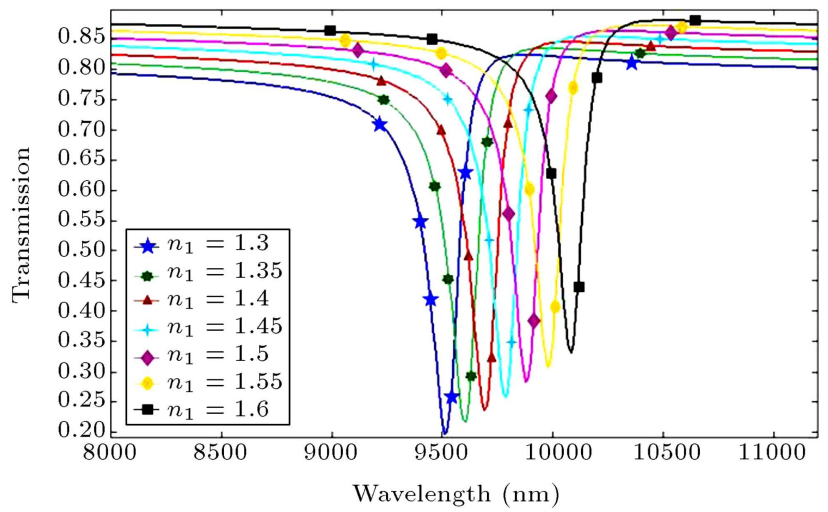

Figure 2. Simulated normal-incidence transmission spectra for different refractive indices of sample medium $n_{1}$ for $E_{f}=0.64 \mathrm{eV}$ and $T=150 \mathrm{~nm}$.

Figure 2 illustrates simulated normal-incidence transmission spectra for different refractive indices of sample medium $\left(n_{1}\right)$ for $E_{f}=0.64 \mathrm{eV}$ and $T=$ $150 \mathrm{~nm}$. It clearly shows that by a small variation in the refractive index, the resonant frequency for which transmission is minimal varies. According to Eq. (2) and $\varepsilon_{r 1}=\left(n_{1}-i k_{1}\right)^{2}$ and considering that $k_{1}=0$, it can be concluded that the resonant frequency $\left(\omega_{0}\right)$ is inversely proportional to cover medium refractive index $\left(n_{1}\right)$. Thus, as shown in Figure 3, the resonant wavelength has a linear relationship with the cover medium refractive index $[19,20]$.

The spectral shift per Refractive Index Unit (RIU) change is proportional to the fraction of the fields and energy density associated with the plasmon excitation located in the sensing medium. Taking the wavelength interrogation approach, sensitivity is defined as the shift of the resonant wavelength as a function of the refractive index of the sample medium $(S=$ $\left.\lambda_{0} / \mathrm{RIU}\right)$. Another major factor in biosensing application is Figure Of Merit (FOM). FOM is considered as a useful parameter in verifying plasmonic biosensor. It is defined as the ratio of the refractive index sensitivity to the resonant width $\left(\mathrm{FOM}=S\left(\mathrm{~nm} \cdot \mathrm{RIU}^{-1}\right) \Delta \lambda(\mathrm{nm})\right)$.

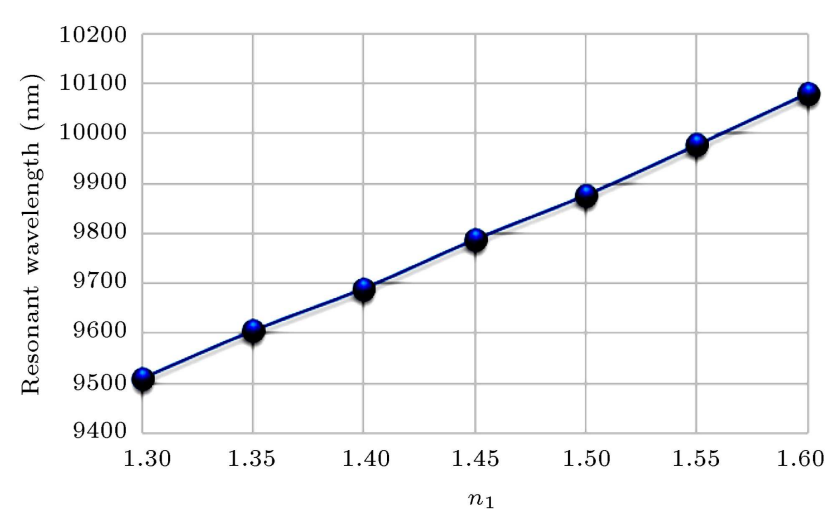

Figure 3. Resonant wavelength of the surface plasmonic wave versus refractive index of the biological sample. 
In most biosensing mechanisms, Biomolecular Recognition Element (BRE) is used to absorb presented analyte or biomolecule in aqueous solution [21]. In this work, we consider antibodies as BRE. By immobilization of the antibodies on the graphene surface, the analytes can be captured by antibodies and form a thin layer on the graphene surface, which induces a local increase in the refractive index near the graphene surface. The schematic mechanism is shown in Figure 4. At the initial stage, when the cover medium only includes the aqueous solution (water), the refractive index is equal to 1.332. When the BRE is added to the aqueous solution, the refractive index of the cover medium changes negligibly. In the next stage, biomolecules are captured by the BRE and the cover medium refractive index increases.

Figure 5 shows simulated normal-incidence transmission spectra for three different cases of medium refractive index around the grating structure: 1) air, 2) cover solution-antibody, and 3) antibody-analyte

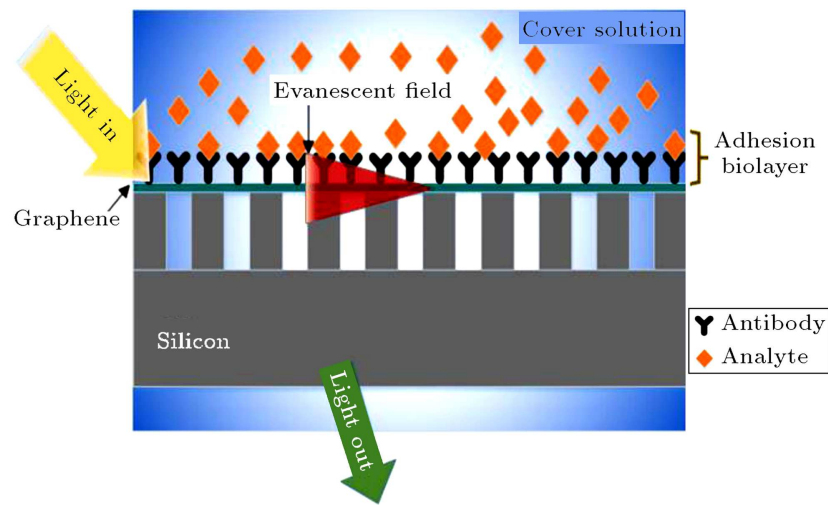

Figure 4. Principle of SPR biosensing; immobilization of the antibodies on the graphene surface to capture the analytes.

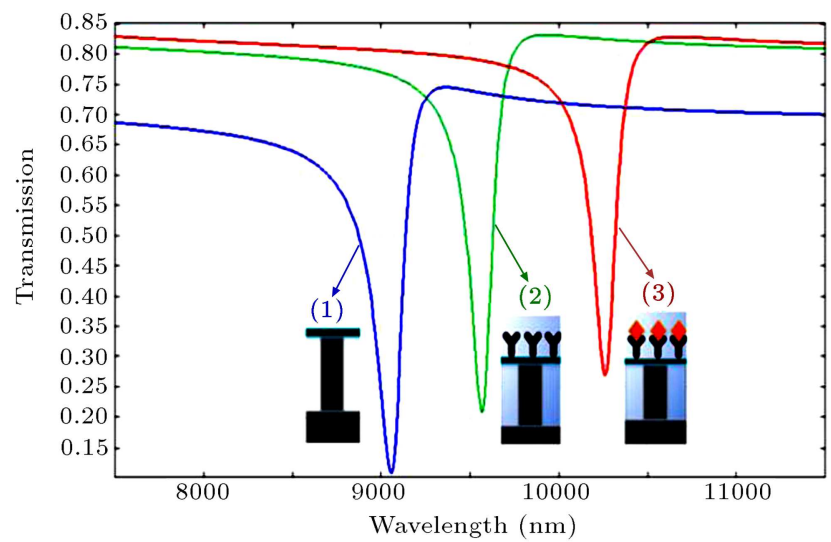

Figure 5. Simulated normal-incidence transmission spectra for three different cases of the medium refractive index changes around the grating structure: 1) air, 2) cover solution-antibody, and 3) antibody-analyte adhesion layer $\left(E_{f}=0.64 \mathrm{eV}\right.$ and $\left.T=150 \mathrm{~nm}\right)$. The thickness of adhesion biolayer is set to $80 \mathrm{~nm}$.

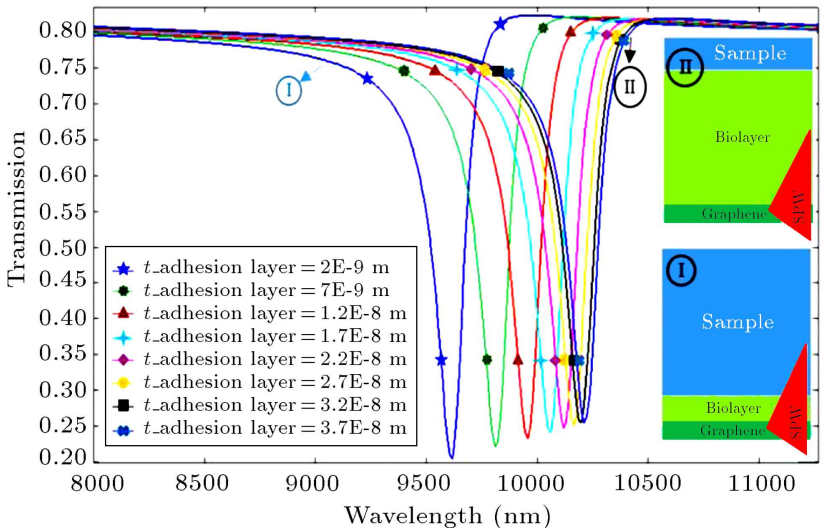

Figure 6. Simulated normal-incidence transmission spectra for different thicknesses of adhesion layer for $E_{f}=0.64 \mathrm{eV}$ and $T=150 \mathrm{~nm}$.

adhesion layer. As shown in Figure 5, before binding the analyte to antibody, the resonant wavelength is $9510 \mathrm{~nm}$ and it is red-shifted to $10272 \mathrm{~nm}$ after binding the analyte, due to the increase in the propagation constant of Surface Plasmon Wave (SPW) propagating along the graphene surface.

The evanescent field of SPW has a limited penetration depth into the graphene adjacent layers. The magnitude of the change in the propagation constant and, consequently, resonant wavelength red-shift of an SPW depends on the refractive index changes (induced by antibody-analyte binding on the graphene surface) and its distribution with respect to the profile of the SPW field and thickness of adhesion layer. As shown in Figure 6, when the refractive index changes are caused by a binding event, which occurs at a specific distance from the sensing surface (much smaller than the penetration depth of the SPW), the magnitude of the resonant wavelength red-shift is large because the whole region of antibody-analyte binding event (the whole thickness of biolayer) is within the extent of the SPW field. By increasing the thickness of adhesion layer, the magnitude of shift in the resonant wavelength is reduced because only a fraction of the thickness of the adhesion layer is within the evanescent field of SPW and depth of the area in which the change occurs is limited (Figure 6). The resonant wavelength change in terms of adhesion layer thickness is shown in Figure 7 .

This sensor has a high spectral shift $\delta \lambda_{0} / \delta n$ with a narrow spectral line width of the plasmon resonance $(\Delta \lambda \approx 1412 \mathrm{~nm})$. The FOM for this plasmonic biosensor structure is approximately equal to 14.2 and the sensitivity exceeds $2000 \mathrm{~nm} / \mathrm{RIU}$, which is larger than most metal mid-IR resonant biosensor structures [22,23]. Recently, a highly sensitive refractive index sensor based on exciting localized Surface Plasmon Polaritons (SPPs) on graphene-coated nanowire arrays has been studied [20]. The sensitivity of this device 


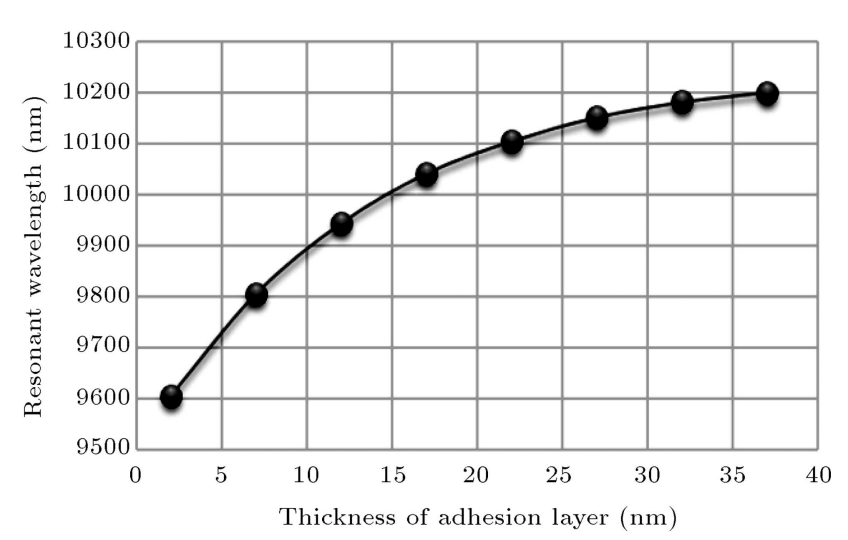

Figure 7. Resonant wavelength of the SPW versus thickness of the adhesion layer.

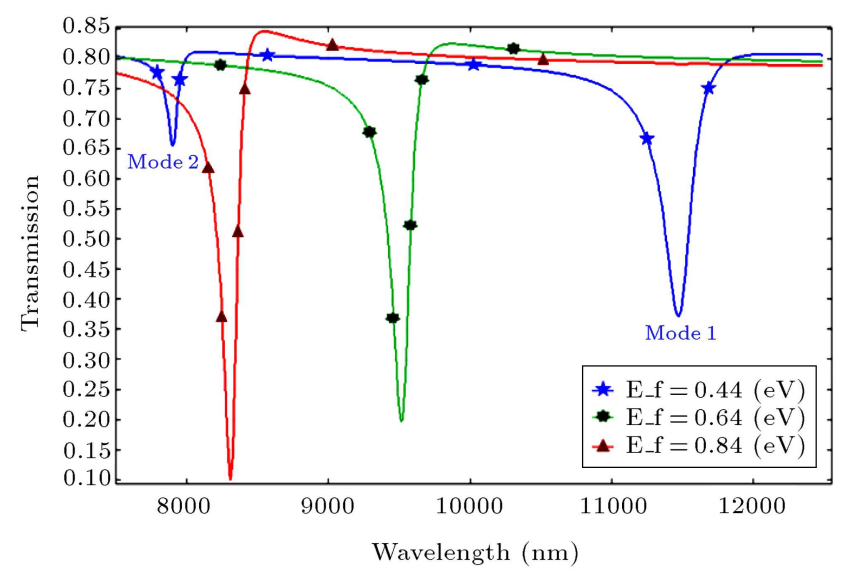

Figure 8. Simulated normal-incidence transmission spectra of monolayer graphene on silicon gratings with different Fermi energy levels in graphene when $n_{1}=1.4$ and $T=150 \mathrm{~nm}$.

is high; however, fabrication process of the proposed structure seems to be difficult. Our proposed structure has some advantages such as simple fabrication process, small size, and easy electrical tuning by applying a gate voltage between the graphene layer and substrate.

Electrical tuning of the proposed plasmonic sensor can be achieved by adjusting the Fermi level in graphene. Sharp notches from the plasmonic wave resonant can be seen. One can identify multiple notches on each transmission spectrum, which result from multiple plasmonic modes. As shown in Figure 8, with a small change in the Fermi level, a broad tuning range can be achieved. One can see in Figure 8 that the resonant wavelength is blue-shifted as the Fermi energy level increases from $0.64 \mathrm{eV}$ to $0.84 \mathrm{eV}$.

In Figure 9(a), the electric field profile of the fundamental mode in one grating period and for a biological medium with specific refractive index $\left(n_{1}=\right.$ $1.4)$ is shown. The notches at the lower wavelength are caused by higher order modes. In Figure $9(\mathrm{~b})$, the electrical field profile of the second-order mode is shown

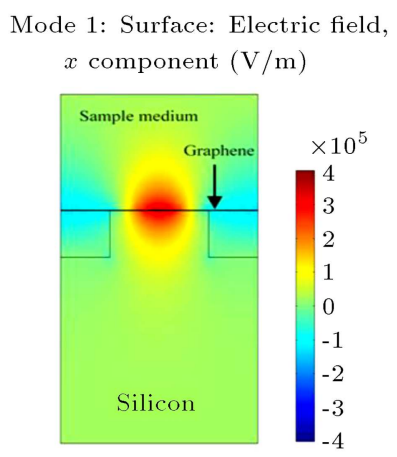

(a)

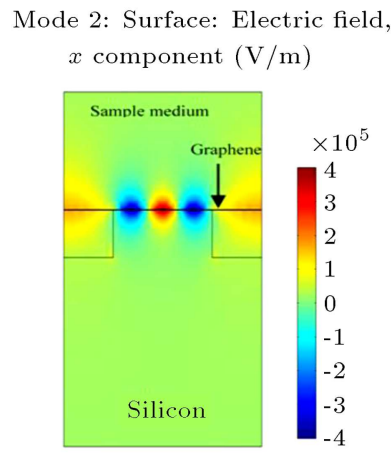

(b)
Figure 9. Side-view of electrical field distribution for (a) the fundamental mode and (b) the second-order mode in one grating period.

where the E-field has a $4 \pi$ phase shift in each grating period.

\section{Conclusions}

We have presented a novel concept for a biological sensor using surface plasmon waves based on periodic diffractive grating structures. This device has a number of interesting benefits. First, due to using periodic diffractive grating structures, the plasmonic wave is highly confined in monolayer graphene films. Second, the device is highly sensitive to the binding events and, consequently, refractive index change of the biological medium. Third, the device is highly tunable due to change in the graphene Fermi level by applying a gate voltage. The numerical simulations show that the proposed structure has a great potential to be used in biosensing applications. The sensitivity for this plasmonic biosensor structure exceeds $2000 \mathrm{~nm} / \mathrm{RIU}$, which is larger than those for most metal mid-IR resonant biosensor structures.

\section{Acknowledgment}

The authors acknowledge the partial support of this project by Iran Nanotechnology Initiative Council (INIC).

\section{References}

1. Allen, M.J., Tung, V.C. and Kaner, R.B. "Honeycomb carbon: A review of graphene", Chem. Rev., 110(1), pp. 132-145 (2010).

2. Geim, A.K. and Novoselov, K.S. "The rise of graphene", Nat. Mater., 6(3), pp. 183-191 (2007).

3. Jablan, M., Buljan, H. and Soljacic, M. "Plasmonics in graphene at infrared frequencies", Phys. Rev. B, 80(24), p. 245435 (2009).

4. Gao, W., Shu, J., Qiu, C. and Xu, Q. "Excitation 
of plasmonic waves in graphene by guided-mode resonants", ACS Nano, 6(9), pp. 7806-7813 (2012).

5. Koppens, F.H.L., Chang, D.E. and de Abajo, F.J.G. "Graphene plasmonics: A platform for strong lightmatter interactions", Nano Lett., 11(8), pp. 3370-3377 (2011).

6. Homola, J. "Surface plasmon resonance sensors for detection of chemical and biological species", Chem. Rev., 180(2), pp. 462-493 (2008).

7. Abdulhalim, I., Zourob, M. and Lakhtakia, A. "Surface plasmon resonance for biosensing: A mini-review", Electromagnetics, 28(3), pp. 214-242 (2008).

8. McGaughey, G.B., Gagné, M. and Rappé, A.K. " $\pi$ stacking interactions. Alive and well in proteins", $J$. Biol. Chem., 273(25), pp. 15458-15463 (1998).

9. Tang, Z., Wu, H., Cort, J.R., Buchko, G.W., Zhang, Y., Shao, Y., Aksay, I.A., Liu, J. and Lin, Y. "Constraint of DNA on functionalized graphene improves its biostability and specificity", Small, 6(11), pp. 12051209 (2010).

10. Fotouhi, B., Ahmadi, V. and Faramarzi, V. "Nanoplasmonic-based structures for DNA sequencing", $O p$ tics Letters, 41(18), pp. 4229-4232 (2016).

11. Zhang, J., Zhang, L. and Xu, W. "Surface plasmon polaritons: physics and applications", Journal of Physics D: Applied Physics, 45(11), p. 113001 (2012).

12. Berini, P. "Long-range surface plasmon polaritons", Advances in Optics and Photonics, 1(3), pp. 484-588 (2009).

13. Parsons, J.T., Horwitz, A.R. and Schwartz, M.A. "Cell adhesion: Integrating cytoskeletal dynamics and cellular tension", Nat. Rev. Mol. Cell Biol., 11(9), pp. 633-643 (2010).

14. Verma, R., Gupta, B.D. and Jha, R. "Sensitivity enhancement of a surface plasmon resonance based biomolecules sensor using graphene and silicon layers", Sensors and Actuators B: Chemical, 160(1), pp. 623631 (2011).

15. Wu, L., Chu, H., Koh, W. and Li, E. "Highly sensitive graphene biosensors based on surface plasmon resonance", Opt. Express, 18(14), pp. 14395-14400 (2010).

16. Jahanshahi, P. and Adikan, F.R.M. "Sensitivity enhancement of graphene-based surface plasmon resonance biosensor using germanium nanowires grating", Journal of Medical and Bioengineering, 4(2), pp. 145149 (2015).

17. Xia, S., Zhai, X., Wang, L., Lin, Q. and Wen, S. "Excitation of crest and trough surface plasmon modes in in-plane bended graphene nanoribbons", Opt. Express, 24(1), pp. 427-436 (2016).

18. Falkovsky, L.A. "Optical properties of graphene", $J$. Phys.: Conf. Ser, 129(1), p. 012004 (2008).

19. Xia, S., Zhai, X., Wang, L., Sun, B., Liu, J. and Wen, S. "Dynamically tunable plasmonically induced transparency in sinusoidally curved and planar graphene layers", Opt. Express, 24(16), pp. 17886-17899 (2016).

20. Xia, S.X., Zhai, X., Wang, L.L., Liu, J.P., Li, H.J., Liu, J.Q., Pan, A.L. and Wen, S.C. "Excitation of surface plasmons in graphene-coated nanowire arrays", Journal of Applied Physics, 120(10), p. 103104 (2016).

21. Homola, Jiří. "Present and future of surface plasmon resonance biosensors", Analytical and Bioanalytical Chemistry, 377(3), pp. 528-539 (2003).

22. Verellen, N., Van Dorpe, P., Huang, C., Lodewijks, K., Vandenbosch, G.A., Lagae, L. and Moshchalkov, V.V. "Plasmon line shaping using nanocrosses for high sensitivity localized surface plasmon resonant sensing", Nano Lett., 11(2), pp. 391-397 (2011).

23. Debackere, P., Scheerlinck, S., Bienstman, P. and Baets, R. "A biosensor based on surface plasmon interference", In 2006 Annual Symposium of the IEEE/LEOS Benelux Chapter, pp. 69-72 (2006).

\section{Biographies}

Vahid Ahmadi (M'86, SM'09) received the PhD degree in Electronic Engineering from the Kyoto University, Japan, in 1994. He is a Professor in Electronic Engineering at Tarbiat Modares University (TMU), Tehran, Iran. He was the Head of the Semiconductor Department of Laser Research Centre, Tehran, during 1994-2006, and the Head of the Electrical Engineering Department of TMU during 2006-2008. He was also the Technical and Scientific Chair of 13th Iranian Conference on Optics and Photonics, 2007, and the general Chair of the 16th Iranian Conference on Electrical Engineering (ICEE2008), 2008. He is the member of the Founders-Board of Optics and Photonics Society of Iran and an editorial board member of International Journal of Information \& Communication Technology Research (IJICTR). He is also the Editor-in-Chief of Modares Journal of Electrical Engineering. His current research interests include quantum photonics devices, CNT and GNR based photonic devices, optical modulator and amplifiers, optical microresonator active and passive devices, all optical switches, slow light, photonic crystals, and plasmonic and metamaterial devices.

Vahid Faramarzi received the BSc degree in Electrical Engineering from Razi University, Kermanshah, Iran, in 2010, and MSc degree in Electrical Engineering from the Department of Electrical Engineering at Sharif University of Technology, Tehran, Iran, in 
2012. He is currently working toward the $\mathrm{PhD}$ degree in the same field with Tarbiat Modares University, Tehran, Iran. His current research interests include biophotonics, optoelectronic devices, and graphene-based sensors.

Farzin Ghane Golmohamadi received his MSc degree from Tarbiat Modares University, Tehran, Iran, in 2012. His research interest is optoelectronic devices used in medical applications. He is currently Head of the Research and Development Department of MehrOptics Company.

Bashir Fotouhi was born in Sanandaj, Iran, in 1988. He received MSc degree in Electrical Engineering from Tarbiat Modares University (TMU), in 2012, and is currently working toward his $\mathrm{PhD}$ degree with TMU, since 2012. His professional interests focus on optoelectronics, bio-photonics, graphene plasmonics, nanopore DNA sequencing, and pattern recognition. His current projects include graphene plasmonics and nanopore DNA sequencing. 